\title{
dEs and the dI fundamental plane
}

\section{Ovidiu Vaduvescu and Marshall L. McCall}

\author{
Department of Physics and Astronomy, York University \\ 128 Petrie Science Building, 4700 Keele St., Toronto, Ontario M3J 1P3, Canada \\ email: ovidiuv@yorku.ca, mccall@yorku.ca
}

\begin{abstract}
Despite much work, the connection between dwarf elliptical galaxies (dEs) and dwarf irregulars (dIs) remains unclear. Recently, we found that the surface brightness profiles (SBPs) of dIs in the near-infrared can be well fitted with a hyperbolic secant (sech) function. From sech fits, a tight relationship was derived between the absolute magnitude, central surface brightness, and $21 \mathrm{~cm}$ line widths, which amounts to a fundamental plane for dIs. Here we examine how closely dEs fit into the dI fundamental plane using published data for $22 \mathrm{dEs}$ in the Virgo cluster and the Local Group. Over a 9 mag interval in absolute magnitude the dEs fall in the plane defined by the dIs. The outstanding overlap suggests more than a casual relationship between the two classes.
\end{abstract}

\section{The dI Sample}

A sample of 19 field dIs with very accurate distances was observed at CFHT adopting a prudent approach to sample the faint outer regions (Vaduvescu and McCall 2004). We separated resolved and unresolved components using the KILLALL package (Buta and McCall 1999) and then derived the surface brightness profiles (SBPs) by fitting ellipses using the stsdas/ellipse function in IRAF. The hyperbolic secant function (sech) was found to fit accurately all the SBPs in our dI sample (Vaduvescu et al. 2005):

$$
I=I_{0} \operatorname{sech}\left(r / r_{0}\right)=\frac{2 I_{0}}{e^{r / r_{0}}+e^{-r / r_{0}}}
$$

where $I_{0}$ is the central surface brightness and $r_{0}$ the scale length. Note that the sech function becomes exponential at large radius.

\section{The dE Sample}

The sample of dEs consists of 22 objects with published data in three sub-samples: 11 dEs in the Virgo Cluster with $\mathrm{H}$ band images from the GOLDMine database (Gavazzi et al. 2002) and velocity dispersions from Geha et al. (2003); three dEs/dSphs in the Local Group (M 110, NGC 147, NGC 185) with K images from 2MASS Large Galaxy Atlas (Jarett et al. 2003) and velocity dispersions from McElroy (1995) and Held et al. (1992); 8 dSphs orbiting our Galaxy (Carina, Draco, Fornax, Leo I, Leo II, Sculptor, Sextans, Ursa Minor) for which star counts in V define surface brightness profiles (Irwin and Hadzidimitriou 1995). We adopted average colors of $\mathrm{H}-\mathrm{K}=0.2 \mathrm{mag}$ and $\mathrm{V}-\mathrm{K}=2.7 \mathrm{mag}$. In all cases, an exponential model was adopted to fit the outer isophotes: $I=I_{0} e^{-r / r_{0}}$.

\section{The Tully-Fisher Relation and the Fundamental Plane}

In Figure 1 (left), we plot the Tully-Fisher relation for both the dIs and dEs with $W_{20}$ estimated for dEs from stellar velocity dispersions. It shows considerable scatter. 
In Figure 1 (right), we show the fundamental plane defined by the absolute magnitude $M_{K}$, the central surface brightness $m_{0}$ derived from $I_{0}$, and the HI line-width $W_{20}$. For the dIs, the "absolute magnitude" $M_{K}$ is the sech magnitude, while for dEs it is the exponential magnitude. The dIs and dEs are marked by crosses and circles, respectively. No corrections for tilt have been applied. The dotted line represents the fit to the dIs alone (defined by the abscissa). The dI fundamental plane shows a remarkable good correlation with a coefficient of correlation 0.91 and a rms scatter of only 0.4 mag. This is comparable to the scatter in the I-band Tully-Fisher relation for spirals adopted in the HST Key Project on the Extragalactic Distance Scale. Over a 9 mag interval in absolute magnitude, the dEs fall in the dI fundamental plane. The outstanding overlap suggests a common scenarion for the early development of the dEs and dIs.
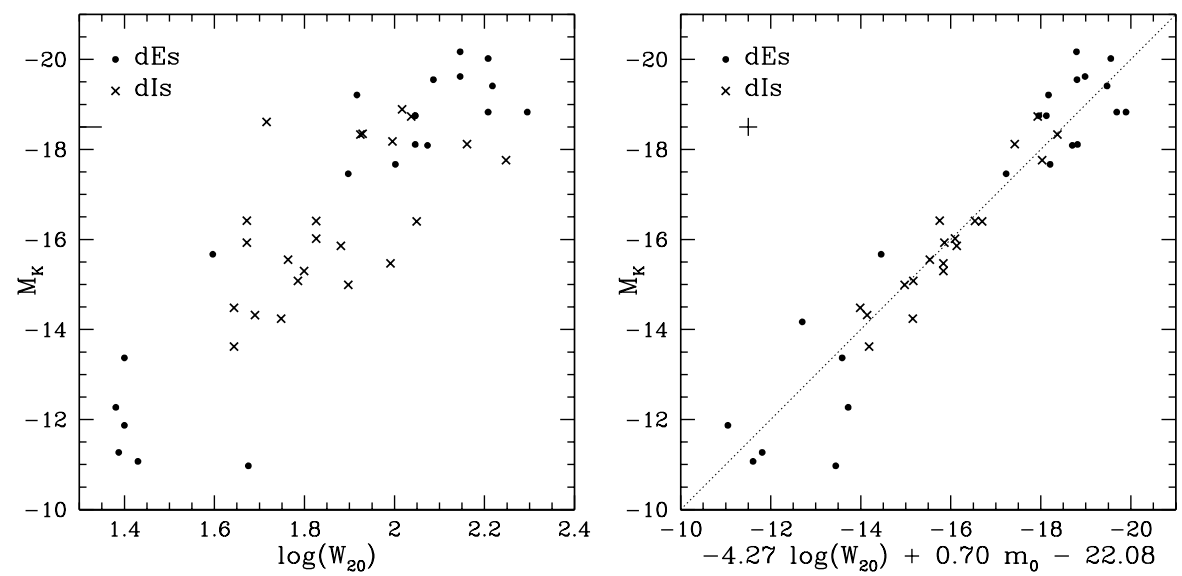

Figure 1. Left: The Tully-Fisher relation appears to hold for both dIs and dEs, although with much scatter. Right: The Fundamental Plane obtained by fitting HI line-widths $W_{20}$, central brightness $m_{0}$, and the sech absolute magnitude $M_{K}$ for $19 \mathrm{dIs}$ with available data (Vaduvescu et al. 2005). The dEs lie on the same plane. A common distance modulus $\mathrm{DM}=30.62$ has been applied for Virgo members. Typical uncertainties are plotted as an error cross.

\section{Acknowledgements}

We are grateful to the Natural Sciences and Engineering Research Council of Canada for its continuing support to the PhD Thesis of OV (2005, in preparation). This research has made use of the GOLDMine Database (Gavazzi et al. 2002) and data products from the Two Micron All Sky Survey. OV would like to thank to Stefano Zibetti for some feedback related to data reduction of the GOLDMine images.

\section{References}

McCall, M.L. \& Buta, R.J. 1999, AJ 109, 6, 2460

Gavazzi, G. et al. 2002, AA 400, 451

Geha, M., Guhathakurta, P. \& van der Marel, R.P. 2003, AJ 103, 3, 851

Held, E.V. et al. 1992, AJ 103, 3, 851

Irwin, M. \& Hadzidimitriou, D. 1995, MNRAS 277, 1354

Jarett, T.H. et al. 2003, AJ 125, 525

McElroy, D.B. 1995, ApJS 100, 105

Vaduvescu, O. \& McCall, M.L. 2004, PASP 116, 820

Vaduvescu, O., McCall, M.L., Richer, M.G. \& Fingerhut, R.L. 2005, AJ, submitted 